\title{
Modeling 2-D AR Processes with Various Regions of Support
}

\author{
Dimitris N. Politis and ByoungSeon Choi *
}

\begin{abstract}
We show that there exists a causal 2-D linear process in the nonsymmetric half-plane having the same autocorrelations as a noncausal 2-D linear process in the whole-plane; this property is called the autocorrelation equivalence relation, and can be used for practical fitting and modeling of 2-D processes. Some causal 2-D AR models with various regions of support are considered such as half-cross, half-diamond, quarter-plane square, half-square, half-hexagon, half-octagon, and halfcircle. Considerations of parsimony in 2-D model fitting are then focused not only on the number of parameters in our model, but also most importantly on the optimal shape of the region of support. Their 2-D Yule-Walker equations are derived, and a computationally efficient order-recursive algorithm is proposed to solve them. The autocorrelation equivalence relation and the order-recursive algorithm are utilized to specify a noncausal 2-D AR process as well as its spectrum from a given realization of a random field.
\end{abstract}

Index Terms: Random field, 2-D AR model, Yule-Walker equations, causal, noncausal, autocorrelation equivalence relation, 2-D spectrum.

*Department of Mathematics, University of California, San Diego, CA 92093, (NSF grant number DMS-01-04059) 


\section{Introduction}

Two-dimensional (2-D) autoregressive (AR) models have many applications in image processing and analysis. For instance, they have been applied to image restoration [1], [2], to texture analysis [3], [4], [5], [6], to fine arts painting analysis [7], and to 2-D spectrum estimation [8], [9], [10]. Let $\left\{y_{s, t}\right\}$ be a second-order stationary random field satisfying a noncausal 2-D AR model

$$
y_{s, t}=\sum_{(j, k) \in D} \beta_{j, k} y_{s-j, t-k}+v_{s, t},
$$

where $\left\{v_{s, t}\right\}$ is a 2-D white noise process with variance $\sigma^{2}>0$. The 2-D index set $D$ can be arbitrary as long as $(0,0)$ does not belong to it, and it is called the region of support (ROS) of the 2-D AR model. Define the autocovariance function (ACVF) and the autocorrelation function (ACRF) of $\left\{y_{s, t}\right\}$ by $\sigma(j, k)=\operatorname{Cov}\left(y_{s+j, t+k}, y_{s, t}\right)$ and $\rho(j, k)=\sigma(j, k) / \sigma(0,0)$, respectively. Since the ACRF is symmetric about the origin, i.e., $\rho(j, k)=\rho(-j,-k)$ for $j, k=0, \pm 1, \pm 2, \cdots$, it is assumed that $D$ and $\left\{\beta_{j, k}\right\}$ are also symmetric about 0 , i.e., $(-j,-k) \in D$ if and only if $(j, k) \in D$, and

$$
\beta_{j, k}=\beta_{-j,-k}, \quad((j, k) \in D)
$$

otherwise, a noncausal 2-D AR process is not identifiable [11], [12, p. 329]. A noncausal 2-D AR model satisfying (2) is said to be symmetric. Define $Q_{p}$ and $H_{p}$, respectively, by $Q_{p}=\{(j, k) \mid j, k=0,1, \cdots, p\} \backslash\{(0,0)\}$ and

$$
H_{p}=\{(j, k) \mid j=1,2, \cdots, p, k=0, \pm 1, \cdots \pm p\} \cup\{(0, k) \mid k=1,2, \cdots, p\} .
$$


Clearly, $H_{p}$ is a subset of the nonsymmetric half-plane (NSHP) $H_{\infty}$ in Fig 1.1 , and $Q_{p}$ is a subset of the first quarter-plane (QP) $Q_{\infty}$ in Fig 1.2. Consider a $2-\mathrm{D}$ AR model with a $\operatorname{ROS} G_{p}\left(\subset H_{\infty}\right)$

$$
y_{s, t}=\sum_{(j, k) \in G_{p}} \beta_{j, k} y_{s-j, t-k}+v_{s, t},
$$

where $\left\{v_{s, t}\right\}$ is a 2 -D white noise process with variance $\sigma^{2}>0$. Since $G_{p}$ is a subset of $H_{\infty}$, a 2-D AR process satisfying (3) is causal.
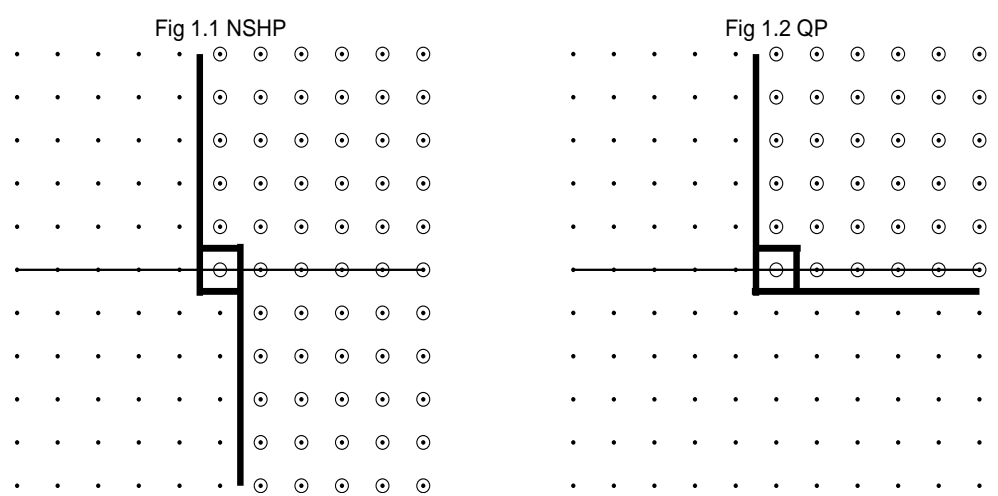

There exist a lot of difficulties in mathematical and statistical analysis of a noncausal 2-D AR process due to its noncausality. Some least squares (LS) estimators and algorithms are proposed in [13], [14], [15], [16]. An LS estimator of an AR coefficient of a noncausal AR model is statistically inferior. To show this, consider a noncausal 1-D AR process satisfying

$$
y_{t}=\theta\left(y_{t-1}+y_{t+1}\right)+v_{t},
$$

where $|\theta|<1 / 2$, and $\left\{v_{t}\right\}$ is a 1 -D white-noise process with variance $\sigma^{2}>0$. It can be verified that $\sigma(k)=\theta\{\sigma(k-1)+\sigma(k+1)\}+\sum_{a=0}^{\infty} \sum_{b=0}^{\infty} \theta^{a}\left(\begin{array}{l}a \\ b\end{array}\right) \sigma(k+a-2 b), \quad(k=0, \pm 1, \pm 2, \cdots)$, 
which are different from the normal equations

$$
\sigma(k)=\theta\{\sigma(k-1)+\sigma(k+1)\}, \quad(k=0, \pm 1, \pm 2, \cdots) .
$$

Therefore, an LS estimator of $\theta$ is biased. Moreover, it is inconsistent and asymptotically inefficient [17]. An LS estimator of a noncausal 2-D AR model is proposed under additive separability assumption of a white-noise process [18]. Also, a maximum likelihood (ML) estimator is proposed under double periodicity assumption [1], [8], [9].

In this paper, it is shown that there exists a causal 2-D linear process in the NSHP that has the same autocorrelations as a symmetric noncausal 2-D linear process. This property is called the autocorrelation equivalence relation (AER) of 2-D linear processes. In addition, causal 2-D AR models with various ROS are considered such as half-cross, half-diamond, QP-square, half-square, half-hexagon, half-octagon, and half-circle. Their 2-D YuleWalker equations are derived, and an order-recursive algorithm is presented to solve them, which is computationally efficient and can be easily implemented as a computer program. Using the AER and the order-recursive algorithm, we can easily specify a noncausal 2-D AR model from its realization. Applications in 2-D spectral analysis are also given.

\section{Autocorrelation Equivalence Relation}

Consider a noncausal 2-D linear process

$$
y_{s, t}=\psi\left(B_{1}^{-1}, B_{2}^{-1}\right) v_{s, t},
$$


where $\left\{v_{s, t}\right\}$ is a 2-D white noise process with variance $\sigma^{2}>0, B_{1}$ and $B_{2}$ are backshift operators satisfying $B_{1}^{j} B_{2}^{k} y_{s, t}=y_{s-j, t-k}$, and

$$
\psi\left(z_{1}, z_{2}\right)=\sum_{a=-\infty}^{\infty} \sum_{b=-\infty}^{\infty} \psi_{a, b} z_{1}^{-a} z_{2}^{-b} .
$$

Assume that the 2-D linear process is stable, i.e.,

$$
\sum_{a=-\infty}^{\infty} \sum_{b=-\infty}^{\infty}\left|\psi_{a, b}\right|<\infty
$$

By the same reason as (2), we assume that

$$
\psi_{j, k}=\psi_{-j,-k}, \quad(j, k=0, \pm 1, \pm 2, \cdots)
$$

Since $v_{s, t}$ is correlated with all the $y_{a, b},(a, b=0, \pm 1, \pm 2 \cdots),\left\{v_{s, t}\right\}$ cannot be regarded as innovations. It results in many difficulties of analyzing a noncausal 2-D linear process. However, they can be overcome through a 2-D polynomial factorization of $\psi\left(z_{1}, z_{2}\right)$ as follows.

Assume that $\psi(1,1)>0$. It it shown $([19]$, p. 1419) that this assumption and the stability assumption imply $\psi\left(z_{1}, z_{2}\right)>0$ on the unit bicircle $\left\{\left(z_{1}, z_{2}\right)|| z_{1}|=| z_{2} \mid=1\right\}$, there is no ambiguity in specifying $\log \left\{\psi\left(z_{1}, z_{2}\right)\right\}$ on the unit bicircle. Define $\left\{\hat{\psi}_{a, b}\right\}$ by

$$
\log \left\{\psi\left(z_{1}, z_{2}\right)\right\}=\sum_{a=-\infty}^{\infty} \sum_{b=-\infty}^{\infty} \hat{\psi}_{a, b} z_{1}^{-a} z_{2}^{-b}
$$

Assumption (10) implies that $\hat{\psi}_{-j,-k}=\hat{\psi}_{j, k},(j, k=0, \pm 1, \pm 2, \cdots)$. A cepstrum $\hat{\gamma}_{j, k}$ is defined [12, pp. 198-208] by

$$
\hat{\gamma}_{j, k}= \begin{cases}\hat{\psi}_{j, k}, & \left((j, k) \in H_{\infty}\right) \\ \hat{\psi}_{0,0}, & ((j, k)=(0,0)), \\ 0, & \text { (otherwise) }\end{cases}
$$


Let $c=\exp \left(\hat{\psi}_{0,0}\right)$ and $\gamma\left(z_{1}, z_{2}\right)=\exp \left(\sum_{(a, b) \in H_{\infty}} \hat{\gamma}_{a, b} z_{1}^{-a} z_{2}^{-b}\right)$. Then, $\psi\left(z_{1}, z_{2}\right)$ satisfies a 2 -D polynomial factorization

$$
\psi\left(z_{1}, z_{2}\right)=c \gamma\left(z_{1}, z_{2}\right) \gamma\left(z_{1}^{-1}, z_{2}^{-1}\right)
$$

We expand $\gamma\left(z_{1}, z_{2}\right)$ as

$$
\gamma\left(z_{1}, z_{2}\right)=-\gamma_{0,0}-\sum_{(a, b) \in H_{\infty}} \gamma_{a, b} z_{1}^{-a} z_{2}^{-b}, \quad \gamma_{0,0}=-1 .
$$

It can be verified using the fundamental theorem of algebra for 2-D polynomials [20] that there exists a unique 2 -D polynomial $\gamma\left(z_{1}, z_{2}\right)$ satisfying a minimum-phase condition. Furthermore, it can be verified using the same method as in [19] that $\gamma_{j, k}$ is real for $(j, k) \in H_{\infty}$, and that $c$ is positive. Equation (13) implies the spectrum of the symmetric noncausal 2-D linear process $(7)$ is

$$
S_{y}\left(\lambda_{1}, \lambda_{2}\right)=c^{2} \frac{\sigma^{2}}{(2 \pi)^{2}}\left|\gamma^{2}\left(e^{i \lambda_{1}}, e^{i \lambda_{2}}\right)\right|^{2} .
$$

Clearly, $S_{y}\left(\lambda_{1}, \lambda_{2}\right)$ in (15) is also the spectrum of a causal 2-D linear process in the NSHP satisfying

$$
y_{s, t}=\gamma^{2}\left(B_{1}^{-1}, B_{2}^{-1}\right) u_{s, t}
$$

where $\left\{u_{s, t}\right\}$ is a 2-D white noise process with variance $c^{2} \sigma^{2}$. Thus, the symmetric noncausal 2-D linear process (7) and the causal 2-D linear process in the NSHP (16) have the same ACRF. This is the autocorrelation equivalence relation (AER) of 2-D linear processes. The corresponding causal 2-D linear process in the NSHP (16) is stable due to its minimum-phase property.

Clearly, it is more convenient and more efficient to handle a causal 2D linear process than a noncausal one. Therefore, the AER of 2-D linear processes is a powerful tool to analyze noncausal 2-D linear processes. 


\section{Causal 2-D AR models with various ROS}

\subsection{The 2-D Yule-Walker equations}

A causal 2-D AR process with a $\operatorname{ROS} G_{p}\left(\subset H_{\infty}\right)$ satisfies

$$
\beta\left(B_{1}^{-1}, B_{2}^{-1}\right) y_{s, t}=v_{s, t}
$$

where $\left\{v_{s, t}\right\}$ is a 2-D white noise process with variance $\sigma^{2}>0$, and

$$
\beta\left(z_{1}, z_{2}\right)=-\beta_{0,0}-\sum_{(a, b) \in G_{p}} \beta_{a, b} z_{1}^{-a} z_{2}^{-b}, \quad \beta_{0,0}=-1
$$

Assume it satisfies a stability condition [20], [21]. Then, the causal 2-D AR process can be represented by

$$
y_{s, t}=v_{s, t}+\sum_{(a, b) \in H_{\infty}} \psi_{a, b} v_{s-a, t-b}
$$

where $\sum_{(a, b) \in H_{\infty}}\left|\psi_{a, b}\right|<\infty$. It can be verified using (19) that the causal 2-D AR process satisfies the 2-D Yule-Walker equations

$$
\rho(j, k)=\sum_{(a, b) \in G_{p}} \beta_{a, b} \rho(j-a, k-b), \quad\left((j, k) \in G_{p}\right) .
$$

The variance of the $2-\mathrm{D}$ white noise process $\left\{v_{s, t}\right\}$ satisfies

$$
\sigma^{2}=\sigma(0,0)\left\{1-\sum_{(a, b) \in G_{p}} \beta_{a, b} \rho(a, b)\right\} .
$$

\subsection{Various ROS}

The principle of parsimony says that it is better to use a smaller number of parameters as far as a selected statistical model is suitable to represent the underlying phenomenon. Statistically speaking, abundant parameters 
result in unreliable parameter estimators. Numerically speaking, they need more calculation than necessary. Hence, a model with few parameters is efficient and desirable. The literature on selecting the number of parameters in time series models using criteria such as AIC and BIC is already quite large; see $[22]$ for a review. By contrast, little is known in the 2-D case. Notably, in the 2-D case the question is not only the number of parameters but also the shape of ROS. We consider causal 2-D AR models with various ROS such as half-cross, half-diamond, QP-square, half-square, half-hexagon, half-octagon, and half-circle, and choose one of the most suitable ROS to a given realization among them.

Define $\left\{g_{n} \mid n=0,1, \cdots, p\right\}$ by $g_{n}=G_{n} \backslash G_{n-1}$ for $n=1,2, \cdots, p$ with $g_{0}=\{(0,0)\}$, where $G_{n} \subset H_{\infty}$. Let $N(n)$ and $S(n)$ be cardinalities of $g_{n}$ and $G_{n}$, respectively, and assume that $N(n)>0$ for $n=1,2, \cdots, p$. In addition, denote elements of $g_{n}$ by

$$
g_{n}=\left\{\left(a_{n, 1}, b_{n, 1}\right),\left(a_{n, 2}, b_{n, 2}\right), \cdots,\left(a_{n, N(n)}, b_{n, N(n)}\right)\right\} .
$$

We arrange the elements of $g_{n}$ in a natural order if it exists. If not, we use the lexicographic order $\prec$, i.e., $\left(a_{k}, b_{k}\right) \prec\left(a_{k+1}, b_{k+1}\right)$ if and only if either $a_{k}<a_{k+1}$ or $\left(a_{k}=a_{k+1}\right.$ and $\left.b_{k}<b_{k+1}\right)$.

\subsubsection{Half-cross ROS}

Let $g_{0}=\{(0,0)\}$, and $g_{n}=\{(0, n),(n, 0)\}$ for $n=1,2, \cdots, p$. Then, $G_{p}$ is a half-cross as shown in Fig 3.1. A corresponding random field is said to satisfy a 2-D AR model with a half-cross ROS. 
(Example 3.1) Consider a 2-D AR model with a half-cross ROS

$$
y_{s, t}=\frac{1}{4} y_{s, t-1}+\frac{1}{4} y_{s-1, t}+v_{s, t} .
$$

This model has been considered by Whittle [23] and Besag [24]. It is verified in Appendix that its ACRF is

$$
\rho(j, k)= \begin{cases}F_{1 / 4}(j, k) / F_{1 / 4}(0,0), & (j+k \geq 0), \\ F_{1 / 4}(-j,-k) / F_{1 / 4}(0,0), & (-j-k \geq 0) .\end{cases}
$$

where $j \geq 0$ and $F_{\theta}(j, k)=\sigma^{2} \sum_{a=0}^{\infty}\left(\begin{array}{c}2 a+j+k \\ a+k\end{array}\right) \theta^{2 a+k+j}$.

$\diamond$

\subsubsection{Half-diamond ROS}

Let $g_{0}=\{(0,0)\}$, and $g_{n}=g_{n}^{(1)} \cup g_{n}^{(2)},(n=1,2, \cdots)$ with $g_{n}^{(1)}=\{(0, n),(1, n-1), \cdots,(n-1,1)\}$ and $g_{n}^{(2)}=\{(n, 0),(n-1,-1), \cdots,(1,-n+1)\}$ for $n=1,2, \cdots, p$. Then, $G_{p}$ is a half-diamond as shown in Fig 3.2. A corresponding random field is said to satisfy a 2-D AR model with a half-diamond ROS.

(Example 3.2) Consider a 2-D AR model with a half-diamond ROS

$$
y_{s, t}=\frac{1}{4} y_{s-1, t-1}+\frac{1}{5} y_{s-1, t+1}-\frac{1}{20} y_{s-2, t}+v_{s, t} .
$$

It is verified in Appendix that, for $j \geq 0$,

$$
\rho(j, k)= \begin{cases}\left(\frac{1}{4}\right)^{\left|\frac{j+k}{2}\right|}\left(\frac{1}{5}\right)^{\left|\frac{j-k}{2}\right|}, & (j+k \bmod 2=0 \text { and } j-k \bmod 2=0), \\ 0, & \text { (otherwise) } .\end{cases}
$$
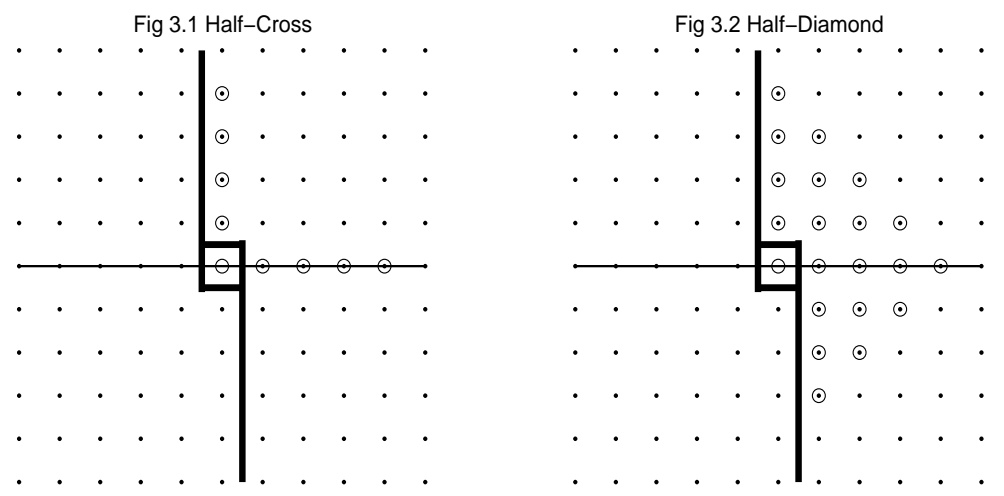
(Example 3.3) Consider a 2-D AR model with a half-diamond ROS

$$
\left(1-\frac{1}{4} B_{1} B_{2}\right)^{2}\left(1-\frac{1}{5} B_{1} B_{2}^{-1}\right)^{2} y_{s, t}=v_{s, t}
$$

It is verified in Appendix that, for $j \geq 0$,

$\rho(j, k)= \begin{cases}\frac{1}{g\left(\frac{1}{4}, 0\right) g\left(\frac{1}{5}, 0\right)} g\left(\frac{1}{4},\left|\frac{j+k}{2}\right|\right) g\left(\frac{1}{5},\left|\frac{j-k}{2}\right|\right), & (j+k \bmod 2=0 \text { and } j-k \bmod 2=0), \\ 0, & \text { (otherwise), }\end{cases}$ where $g(\nu, l)=\frac{\nu^{l+2}}{\left(1-\nu^{2}\right)^{3}}\left\{2+(l-1)\left(1-\nu^{2}\right)\right\}$.

\subsubsection{Quarter-plane square ROS}

Let $g_{0}=\{(0,0)\}$, and $g_{n}=g_{n}^{(1)} \cup g_{n}^{(2)},(n=1,2, \cdots)$ with $g_{n}^{(1)}=\{(0, n),(1, n), \cdots,(n-1, n)\}$ and $g_{n}^{(2)}=\{(n, n),(n, n-1), \cdots,(n, 0)\}$ for $n=1,2, \cdots, p$. Then, $G_{p}$ is a square in the first $\mathrm{QP}$ as shown in Fig 3.3. A corresponding random field is said to satisfy a 2-D AR model with a QP-square ROS.
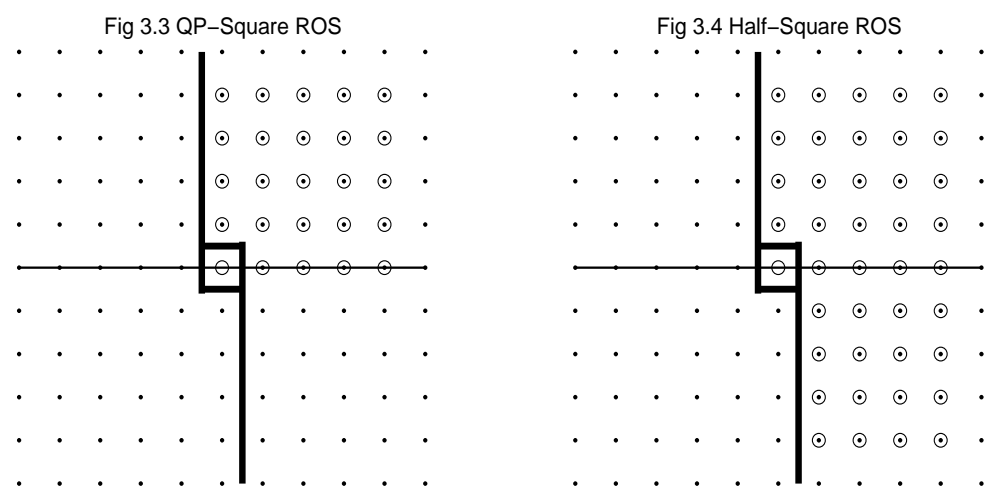

(Example 3.4) Consider a 2-D AR model with a QP-square ROS

$$
y_{s, t}=\frac{1}{4} y_{s, t-1}+\frac{1}{5} y_{s-1, t}-\frac{1}{20} y_{s-1, t-1}+v_{s, t} .
$$

It can be easily verified that

$$
\rho(j, k)=\left(\frac{1}{4}\right)^{|j|}\left(\frac{1}{5}\right)^{|k|}, \quad(j, k=0,1, \cdots) . \quad \diamond
$$




\subsubsection{Half-square ROS}

Let $g_{0}=\{(0,0)\}$, and $g_{n}=g_{n}^{(1)} \bigcup g_{n}^{(2)} \cup g_{n}^{(3)},(n=1,2, \cdots, p)$ with $g_{n}^{(1)}=$ $\{(0, n),(1, n), \cdots,(n-1, n)\}, g_{n}^{(2)}=\{(n, n),(n, n-1), \cdots,(n,-n+1)\}$, and $g_{n}^{(3)}=\{(n,-n),(n-1,-n), \cdots,(1,-n)\}$ for $n=1,2, \cdots, p$. Then, $G_{p}$ is a half-square in the NSHP as shown in Fig 3.4. A corresponding random field is said to satisfy a 2-D AR model with a half-square ROS.

(Example 3.5) Consider a 2-D AR model with a half-square ROS

$$
y_{s, t}=0.2 y_{s-1, t-1}+0.2 y_{s-1, t+1}+v_{s, t} .
$$

It is verified in [19] that

$$
\begin{aligned}
\rho(j, k) & =\rho(j,-k)=\rho(-j, k)=\rho(-j,-k), \quad(j, k \geq 0), \\
\rho(j, k) & =0, \quad(j+k \bmod 2=1 \text { and } j, k \geq 0), \\
\rho(j, k)= & \left(\frac{1-\sqrt{1-4 \theta^{2}}}{2 \theta}\right)^{k}, \quad(j+k \bmod 2=0 \text { and } k \geq j \geq 0), \\
\rho(j, k)= & \left(\frac{1-\sqrt{1-4 \theta^{2}}}{2 \theta}\right)^{k}-\sqrt{1-4 \theta^{2}} \sum_{a=0}^{\frac{j-k}{2}-1}\left(\begin{array}{c}
2 a+k \\
a+k
\end{array}\right) \theta^{2 a+k}, \\
& (j+k \bmod 2=0 \text { and } j>k \geq 0) .
\end{aligned}
$$
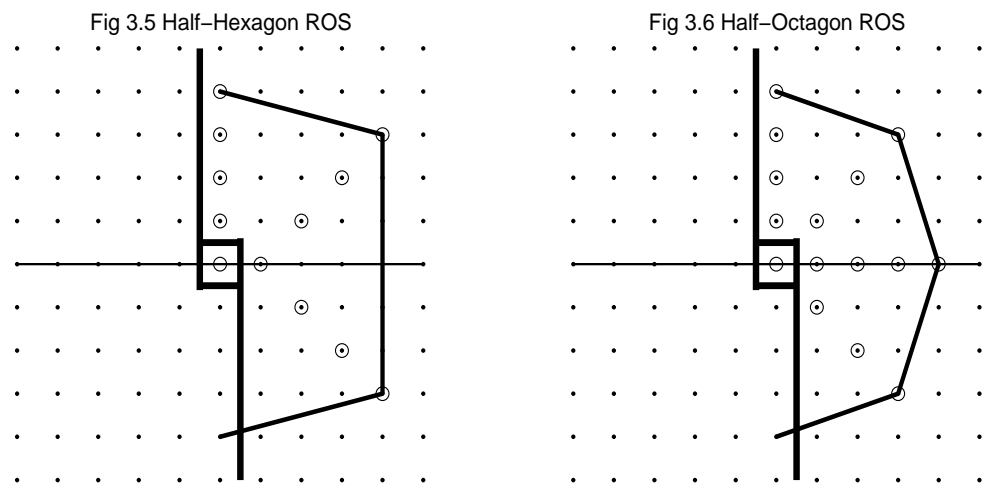


\subsubsection{Half-hexagon ROS}

Let $g_{0}=\{(0,0)\}, g_{1}=\{(0,1),(1,0)\}$, and $g_{n}=\{(0, n),(n, n-1),(n, 1-n)\}$ for $n=2,3, \cdots, p$. Then, $G_{p}$ is a half-hexagon as shown in Fig 3.5. A corresponding random field is said to satisfy a 2-D AR model with a half-hexagon ROS. It should be noted that this $G_{p}$ does not cover all the lattices inside and on $g_{p}$.

(Example 3.6) Consider a 2-D AR model with a half-hexagon ROS

$$
y_{s, t}=\frac{1}{4} y_{s, t-2}+\frac{1}{5} y_{s-2, t+1}-\frac{1}{20} y_{s-2, t-1}+v_{s, t} .
$$

It is verified in Appendix that, for $j \geq 0$,

$$
\rho(j, k)= \begin{cases}\left(\frac{1}{4}\right)^{\left|\frac{j+2 k}{4}\right|}\left(\frac{1}{5}\right)^{\left|\frac{j}{2}\right|}, & (j+2 k \bmod 4=0 \text { and } j \bmod 2=0), \\ 0, & \text { (otherwise) } .\end{cases}
$$

\subsubsection{Half-octagon ROS}

Let $g_{0}=\{(0,0)\}, g_{1}=\{(0,1),(1,0)\}$, and $g_{n}=\{(0, n),(n-1, n-1),(n, 0),(n-1,1-n)\}$ for $n=2,3, \cdots, p$. Then, $G_{p}$ is a half-octagon as shown in Fig 3.6. A corresponding random field is said to satisfy a 2-D AR model with a half-octagon ROS. It is also called a 2-D AR process with a ROS of a fundamental cycle of length $8[25]$. It should be noted that this $G_{p}$ does not cover all the lattices inside and on $g_{p}$.

(Example 3.7) Consider a 2-D AR model with a half-hexagon ROS

$$
y_{s, t}=\frac{1}{4} y_{s, t-2}+\frac{1}{5} y_{s-2, t+2}-\frac{1}{20} y_{s-2, t}+v_{s, t} .
$$

It is verified in Appendix that, for $j \geq 0$,

$$
\rho(j, k)= \begin{cases}\left(\frac{1}{4}\right)^{\left|\frac{j+k}{2}\right|}\left(\frac{1}{5}\right)^{\left|\frac{j}{2}\right|}, & (j+k \bmod 2=0 \text { and } j \bmod 2=0), \\ 0, & \text { (otherwise). }\end{cases}
$$




\subsubsection{Half-circle ROS}

We consider a close-neighbor ordering in the NSHP discussed in [13], [15], $[26]$. Let $g_{0}=\{(0,0)\}$, and

$$
g_{n}=\left\{(a, b) \in H_{\infty} \mid n-1<\sqrt{a^{2}+b^{2}} \leq n\right\}, \quad(n=1,2, \cdots, p) .
$$

We arrange elements of $g_{n}$ in the lexicographic order $\prec$. It is clear that $G_{p}$ is a half-circle in the NSHP as shown in Fig 3.7. A corresponding random field is said to satisfy a 2-D AR model with a half-circle ROS.

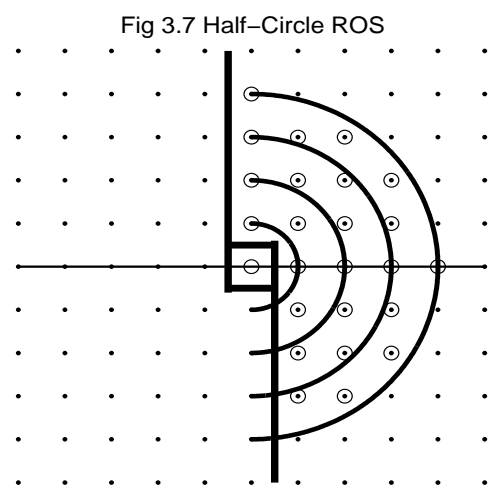

(Example 3.7) Consider a 2-D AR model with a half-circle ROS

$$
y_{s, t}=\frac{1}{4} y_{s, t-3}+\frac{1}{5} y_{s-2, t+2}-\frac{1}{20} y_{s-2, t-1}+v_{s, t} .
$$

It is verified in Appendix that, for $j \geq 0$,

$$
\rho(j, k)= \begin{cases}\left(\frac{1}{4}\right)^{\left|\frac{j+k}{3}\right|}\left(\frac{1}{5}\right)^{\left|\frac{j}{2}\right|}, & (j+k \bmod 3=0 \text { and } j \bmod 2=0), \\ 0, & \text { (otherwise) } .\end{cases}
$$




\subsection{An order-recursive algorithm}

To identify a causal 2-D AR process with a $\operatorname{ROS} G_{p}$ when the ACRF $\{\rho(j, k)\}$ is given, it is necessary to solve the 2-D Yule-Walker equations in (20) and (21) for $p=1,2, \cdots$. To fulfill this purpose, we present an order-recursive algorithm as follows.

For each $m(=1,2, \cdots)$, let $\left\{\beta_{m}(j, k) \mid(j, k) \in G_{m}\right\}$ be a solution of the 2-D Yule-Walker equations

$$
\rho(j, k)=\sum_{(a, b) \in G_{m}} \beta_{m}(a, b) \rho(j-a, k-b), \quad\left((j, k) \in G_{m}\right) .
$$

A corresponding variance of the 2-D white noise process is defined by

$$
\sigma_{G_{m}}^{2}=\sigma(0,0)\left\{1-\sum_{(a, b) \in G_{m}} \beta_{m}(a, b) \rho(a, b)\right\} .
$$

Following the methods of deriving order-recursive algorithms for 2-D AR models with QP-square ROS [27] and 3-D AR models with nonsymmetric half-space ROS [28], we represent the 2-D Yule-Walker equations in (23) and (24) as a matrix form. For notational convenience, let

$$
\begin{aligned}
& \alpha_{m}(0,0)=\frac{\sigma(0,0)}{\sigma_{G_{m}}^{2}}, \\
& \alpha_{m}(j, k)=-\frac{\sigma(0,0)}{\sigma_{G_{m}}^{2}} \beta_{m}(j, k), \quad\left((j, k) \in G_{m}\right) .
\end{aligned}
$$

It is clear that the 2-D Yule-Walker equations in (23) and (24) become

$$
\alpha_{m}(0,0) \rho(j, k)+\sum_{(a, b) \in G_{m}} \alpha_{m}(a, b) \rho(j-a, k-b)= \begin{cases}1, & (j=k=0), \\ 0, & \left((j, k) \in G_{m}\right) .\end{cases}
$$


For each $n(=1,2, \cdots, m)$, define an $N(n)$-dimensional row vector $\boldsymbol{\rho}(j, k ; n)$ and an $N(n)$-dimensional column vector $\boldsymbol{\alpha}_{m}(n)$, respectively, by $\boldsymbol{\rho}(j, k ; n)=\left[\begin{array}{c}\rho\left(j-a_{n, 1}, k-b_{n, 1}\right) \\ \rho\left(j-a_{n, 2}, k-b_{n, 2}\right) \\ \vdots \\ \rho\left(j-a_{n, N(n)}, k-b_{n, N(n)}\right)\end{array}\right]^{t}, \quad \boldsymbol{\alpha}_{m}(n)=\left[\begin{array}{c}\alpha_{m}\left(a_{n, 1}, b_{n, 1}\right) \\ \alpha_{m}\left(a_{n, 2}, b_{n, 2}\right) \\ \vdots \\ \alpha_{m}\left(a_{n, N(n)}, b_{n, N(n)}\right)\end{array}\right]$ with $\boldsymbol{\rho}(j, k ; 0)=[\rho(j, k)]$ and $\boldsymbol{\alpha}_{m}(0)=\left[\alpha_{m}(0,0)\right]$. In addition, let

$$
\boldsymbol{\beta}_{m}(n)=-\frac{\sigma_{G_{m}}^{2}}{\sigma(0,0)} \boldsymbol{\alpha}_{m}(n), \quad(n=1,2, \cdots, m) .
$$

It can be verified that

$$
\begin{aligned}
& \sum_{(a, b) \in G_{m}} \alpha_{m}(a, b) \rho(j-a, k-b) \\
& =\sum_{n=1}^{m} \sum_{(a, b) \in g_{n}} \alpha_{m}(a, b) \rho(j-a, k-b) \\
& =\sum_{n=1}^{m} \sum_{c=1}^{N(n)} \alpha_{m}\left(a_{n, c}, b_{n, c}\right) \rho\left(j-a_{n, c}, k-b_{n, c}\right) \\
& =\sum_{n=1}^{m} \boldsymbol{\rho}(j, k ; n) \boldsymbol{\alpha}_{m}(n) .
\end{aligned}
$$

Combining (27) and (29) shows that the 2-D Yule-Walker equations in (23) and (24) become

$$
\sum_{n=0}^{m} \boldsymbol{\rho}(j, k ; n) \boldsymbol{\alpha}_{m}(n)= \begin{cases}1, & (j=k=0), \\ 0, & \left((j, k) \in G_{m}\right) .\end{cases}
$$

For each pair $(n, l)$ of positive integers, define a $1 \times N(l)$ matrix $R_{0, l}$, an $N(n) \times 1$ matrix $R_{n, 0}$ and an $N(n) \times N(l)$ matrix $R_{n, l}$, respectively, by

$$
\begin{aligned}
& R_{0, l}=\boldsymbol{\rho}(0,0 ; l) \\
& R_{n, 0}=\left[\begin{array}{c}
\boldsymbol{\rho}\left(a_{n, 1}, b_{n, 1} ; 0\right) \\
\boldsymbol{\rho}\left(a_{n, 2}, b_{n, 2} ; 0\right) \\
\vdots \\
\boldsymbol{\rho}\left(a_{n, N(n)}, b_{n, N(n)} ; 0\right)
\end{array}\right], \quad R_{n, l}=\left[\begin{array}{c}
\boldsymbol{\rho}\left(a_{n, 1}, b_{n, 1} ; l\right) \\
\boldsymbol{\rho}\left(a_{n, 2}, b_{n, 2} ; l\right) \\
\vdots \\
\boldsymbol{\rho}\left(a_{n, N(n)}, b_{n, N(n)} ; l\right)
\end{array}\right] .
\end{aligned}
$$


Also, let $R_{0,0}=$ [1]. It can be easily shown that $R_{l, n}^{t}=R_{n, l}$ for $l, n=$ $0,1, \cdots$. Define an $(S(m)+1) \times(S(m)+1)$ matrix $R_{m}$ and an $(S(m)+1)$ dimensional column vector $\boldsymbol{\alpha}_{m}$, respectively, by

$$
R_{m}=\left[\begin{array}{cccc}
R_{0,0} & R_{0,1} & \cdots & R_{0, m} \\
R_{1,0} & R_{1,1} & \cdots & R_{1, m} \\
\vdots & \vdots & & \vdots \\
R_{m, 0} & R_{m, 1} & \cdots & R_{m, m}
\end{array}\right] \text { and } \boldsymbol{\alpha}_{m}=\left[\begin{array}{c}
\boldsymbol{\alpha}_{m}(0) \\
\boldsymbol{\alpha}_{m}(1) \\
\vdots \\
\boldsymbol{\alpha}_{m}(m)
\end{array}\right]
$$

If $j=k=0$, then (30) becomes

$$
\sum_{n=0}^{m} R_{0, n} \boldsymbol{\alpha}_{m}(n)=\sum_{n=0}^{m} \boldsymbol{\rho}(0,0 ; n) \boldsymbol{\alpha}_{m}(n)=1 .
$$

Otherwise, (30) becomes

$$
\sum_{n=0}^{m} R_{a, n} \boldsymbol{\alpha}_{m}(n)=\mathbf{0}, \quad(a=1,2, \cdots, m) .
$$

Combining (31) and (32) results in a matrix representation

$$
R_{m} \boldsymbol{\alpha}_{m}=\boldsymbol{n}_{S(m)+1}
$$

where $\boldsymbol{n}_{k}$ is a $k$-dimensional column vector defined by $\boldsymbol{n}_{k}=(1,0,0, \cdots, 0)^{t}$. Since $R_{m}$ is nonsingular, (33) has a unique solution for each $m(=1,2, \cdots)$.

Since the dimension of each submatrix $R_{a, b}$ of $R_{m}$ is not fixed but depends on $a$ and $b, R_{m}$ is not a block Toeplitz matrix. Thus, we can not apply the same method to solve (33) as block Toeplitz inversion algorithms like [29], [30]. Instead, we solve it recursively using the following algorithm, which is derived through a bordering matrix technique. Applying this algorithm to calculation of $\left\{\beta_{m}(j, k) \mid(j, k) \in G_{m}\right\}$ and $\sigma_{G_{m}}^{2}$ for $m=1,2, \cdots$, we first solve the 2-D Yule-Walker equations in (33) for $\boldsymbol{\alpha}_{1}, \boldsymbol{\alpha}_{2}, \cdots$, and then, obtain $\beta_{m}(j, k)$ and $\sigma_{G_{m}}^{2}$ through (25) and (26).

\section{[ Algorithm A ]}


- Initial stage.

$$
\begin{aligned}
& R^{1,1}=R_{1,1}^{-1}, \quad \boldsymbol{\beta}_{1}(1)=R^{1,1} R_{1,0}, \quad \Phi_{2,1}(0)=R^{1,1} R_{1,2}, \\
& \lambda_{2}=1-R_{0,1} \boldsymbol{\beta}_{1}(1), \quad \sigma_{G_{1}}^{2}=\sigma(0,0) \lambda_{2} .
\end{aligned}
$$

- For $m=2,3, \cdots$, calculate

$$
\begin{aligned}
& \Theta_{m}=R_{m, m}-\sum_{a=1}^{m-1} R_{m, a} \Phi_{m, a}(0), \\
& \boldsymbol{h}_{m}=R_{m, 0}-\sum_{a=1}^{m-1} R_{m, a} \boldsymbol{\beta}_{m-1}(a), \\
& \boldsymbol{\beta}_{m}(m)=\Theta_{m}^{-1} \boldsymbol{h}_{m}, \\
& \boldsymbol{\beta}_{m}(a)=\boldsymbol{\beta}_{m-1}(a)-\Phi_{m, a}(0) \boldsymbol{\beta}_{m}(m), \quad(a=1,2, \cdots, m-1), \\
& \lambda_{m+1}=\lambda_{m}-\boldsymbol{h}_{m}^{t} \boldsymbol{\beta}_{m}(m), \\
& \sigma_{G_{m}}^{2}=\sigma(0,0) \lambda_{m+1}, \\
& \Phi_{2,1}(m-1)=R^{1,1} R_{1, m+1} .
\end{aligned}
$$

For $n=2,3, \cdots, m$, calculate

$$
\begin{gathered}
\Phi_{n+1, n}(m-n)=\Theta_{n}^{-1}\left\{R_{n, m+1}-\sum_{a=1}^{n-1} R_{n, a} \Phi_{n, a}(m-n+1)\right\}, \\
\Phi_{n+1, a}(m-n)=\Phi_{n, a}(m-n+1)-\Phi_{n, a}(0) \Phi_{n+1, n}(m-n), \\
(a=1,2, \cdots, n-1) . \quad \diamond
\end{gathered}
$$

It should be noted that $\Theta_{m}$ is an $N(m) \times N(m)$ matrix, $\Phi_{l, a}(k)$ is an $N(a) \times N(l+k)$ matrix, $\boldsymbol{h}_{m}$ is an $N(m)$-dimensional column vector, and $\boldsymbol{\beta}_{m}(a)$ is an $N(a)$-dimensional column vector. Most of Levinson-type orderrecursive algorithms for 2-D AR models are to solve the 2-D Yule-Walker 
equations of 2-D AR processes with either QP-square ROS or half-square ROS, and they assume one of width and length of a ROS square is fixed. In that case, a corresponding covariance matrix is block-Toeplitz, and an orderrecursive algorithm can be easily derived using a block LU decomposition [29], [30], [22, p. 82] as discussed before. Applying a one-to-one mapping between a 2 -D index set $G_{p}$ and an integer set $\{1,2, \cdots, S(p)\}$ to the 2 -D Yule-Walker equations, we regard the 2-D Yule-Walker equations as 1-D Yule-Walker equations. Then, we use the 1-D Levinson-Durbin algorithm to solve the 2-D Yule-Walker equations. However, it should be noted that only one parameter can be added in each step [26].

(Example 3.9) Let $\left\{\rho(j, k)=\rho(-j,-k) \mid(j, k) \in H_{\infty}\right\}$ be the ACRF of a 2-D AR model with a half-diamond ROS satisfying $\rho(j, k)=\rho(-j,-k)= \begin{cases}\left(\frac{1}{4}\right)^{\left|\frac{k+j}{2}\right|}\left(\frac{1}{5}\right)^{\left|\frac{j-k}{2}\right|}, & (j+k \bmod 2=0, j-k \bmod 2=0), \\ 0, & \text { (otherwise), }\end{cases}$ and let $\sigma^{2}=0.9$. To find a causal 2-D AR model with a half-diamond ROS, we apply Algorithm A to this ACRF. For $m=1$, Algorithm A yields that $\boldsymbol{\beta}_{1}(1)=\left[\begin{array}{ll}0 & 0\end{array}\right]^{t}$ and $\sigma_{G_{1}}^{2}=1.0$. For $m=2$, Algorithm A yields that $\boldsymbol{\beta}_{2}(1)=\left[\begin{array}{ll}0 & 0\end{array}\right]^{t}, \boldsymbol{\beta}_{2}(2)=\left[\begin{array}{llll}0 & 0.2500 & -0.0500 & 0.2000\end{array}\right]^{t}$, and $\sigma_{G_{2}}^{2}=0.9000$. For $m \geq 3$, Algorithm A yields that $\boldsymbol{\beta}_{m}(1)=\left[\begin{array}{ll}0 & 0\end{array}\right]^{t}$, $\boldsymbol{\beta}_{m}(2)=\left[\begin{array}{llll}0.000 & 0.2500 & -0.0500 & 0.2000\end{array}\right]^{t}, \boldsymbol{\beta}_{m}(n)=\mathbf{0}, \quad(n=3,4, \cdots)$, and $\sigma_{G_{m}}^{2}=0.9000$. Thus, the ACRF is from a causal 2-D AR model with a half-diamond ROS

$$
y_{s, t}=\frac{1}{4} y_{s-1, t-1}-\frac{1}{20} y_{s-2, t}+\frac{1}{5} y_{s-1, t+1}+v_{s, t},
$$

where $\left\{v_{s, t}\right\}$ is a $2-\mathrm{D}$ white noise process with variance 0.9000 . It is shown in Appendix that the ACRF is truly from this 2-D AR model. 


\section{Noncausal 2-D AR modeling}

Since the symmetric noncausal 2-D AR process (1) is assumed to be stable, it can be represented by a $2-\mathrm{D}$ AR linear model (7). Thus, there exists a causal 2-D AR process having the same ACRF as a symmetric noncausal 2-D AR process. This relation is called the AER of 2-D AR processes. It is convenient and efficient to specify a noncausal 2-D AR model from its ACRF using the AER and Algorithm A. To illustrate it, we consider the following example.

(Example 4.1) Consider an $\operatorname{ACRF}\left\{\rho(j, k)=\rho(-j,-k) \mid(j, k) \in H_{\infty}\right\}$ of a symmetric noncausal 2-D AR process satisfying

$\rho(j, k)= \begin{cases}\frac{1}{g\left(\frac{1}{4}, 0\right) g\left(\frac{1}{5}, 0\right)} g\left(\frac{1}{4},\left|\frac{j+k}{2}\right|\right) g\left(\frac{1}{5},\left|\frac{j-k}{2}\right|\right), & (j+k \bmod =0 \text { and } j-k \bmod 2=0), \\ 0, & \text { (otherwise), }\end{cases}$

where $g(\nu, l)=\nu^{l+2}\left\{2+(l-1)\left(1-\nu^{2}\right)\right\} /\left(1-\nu^{2}\right)^{3}$, with $\sigma^{2}=0.6597$.

To find a symmetric noncausal 2-D AR model having this ACRF, we apply Algorithm A of causal 2-D AR models with half-diamond ROS to this ACRF.

For each $m(=1,2, \cdots)$, define a $2 m \times(m+1)$ matrix $B_{m}$ by

$$
B_{m}=\left[\begin{array}{ccccc}
\beta_{m}(0, m) & X & X & X & X \\
\beta_{m}(0, m-1) & \beta_{m}(1, m-1) & X & X & X \\
\vdots & \vdots & & & \\
\beta_{m}(0,1) & \beta_{m}(1,1) & \cdots & \beta_{m}(m-1,1) & X \\
\beta_{m}(0,0) & \beta_{m}(1,0) & \cdots & \beta_{m}(m-1,0) & \beta_{m}(m, 0) \\
X & \beta_{m}(1,-1) & \cdots & \beta_{m}(m-1,-1) & X \\
\vdots & \vdots & & & \\
X & \beta_{m}(1,1-m) & \cdots & X & X
\end{array}\right]
$$

where $X$ means 'no element'. If $m=1$, Algorithm A yields that

$$
B_{1}=\left[\begin{array}{ll}
0.0000 & X \\
-1 & 0.0000
\end{array}\right], \quad \sigma_{G_{1}}^{2}=1
$$


If $m=2$, Algorithm A yields that

$$
B_{2}=\left[\begin{array}{lll}
0.0000 & X & X \\
0.0000 & 0.4706 & X \\
-1 & 0.0000 & -.1810 \\
X & 0.3846 & X
\end{array}\right], \quad \sigma_{G_{2}}^{2}=0.6634
$$

If $m=3$, Algorithm A yields that

$$
B_{3}=\left[\begin{array}{llll}
0.0000 & X & X & X \\
0.0000 & 0.0000 & X & X \\
0.0000 & 0.4706 & 0.0000 & X \\
-1 & 0.0000 & -0.1810 & 0.0000 \\
X & 0.3846 & 0.0000 & X \\
X & 0.0000 & X & X
\end{array}\right], \quad \sigma_{G_{3}}^{2}=0.6634
$$

For $m \geq 4$, Algorithm A yields that $\sigma_{G_{m}}^{2}=6597$ and

$$
B_{m}=\left[\begin{array}{lllllll}
0.0000 & X & X & X & X & X & X \\
\vdots & \vdots & & & & & \vdots \\
0.0000 & X & X & X & X & X & X \\
0.0000 & 0.0000 & X & X & X & X & X \\
0.0000 & 0.0000 & -.0625 & X & X & X & X \\
0.0000 & 0.5000 & 0.0000 & 0.0250 & X & X & X \\
-1 & 0.0000 & -0.2000 & 0.0000 & -.0025 & X & X \\
X & 0.4000 & 0.0000 & 0.0200 & X & X & X \\
X & 0.0000 & -.0400 & X & X & X & X \\
X & 0.0000 & X & X & X & X & X \\
& \vdots & & & & & \vdots \\
X & 0.0000 & X & X & X & X & X
\end{array}\right] .
$$

Hence, we can conclude that the given ACRF is from a causal 2-D AR model $\beta\left(B_{1}^{-1}, B_{2}^{-1}\right) y_{s, t}=v_{s, t}$, where $\left\{v_{s, t}\right\}$ is a $2-\mathrm{D}$ white noise process with variance 0.6597 , and

$$
\begin{aligned}
& \beta\left(z_{1}^{-1}, z_{2}^{-1}\right)=1-\frac{1}{2} z_{1}^{-1} z_{2}^{-1}+\frac{1}{5} z_{1}^{-2}-\frac{2}{5} z_{1}^{-1} z_{2}+\frac{1}{16} z_{1}^{-2} z_{2}^{-2}-\frac{1}{40} z_{1}^{-3} z_{2} \\
& +\frac{1}{400} z_{1}^{-4}-\frac{1}{50} z_{1}^{-3} z_{2}+\frac{1}{25} z_{1}^{-2} z_{2}^{2}
\end{aligned}
$$


It is shown in Appendix that the ACRF is truly from this 2-D AR model. It can be verified using Equation (31) of [19] that

$$
\beta\left(z_{1}^{-1}, z_{2}^{-1}\right)=\gamma^{2}\left(z_{1}^{-1}, z_{2}^{-1}\right)=\left(1-\frac{1}{4} z_{1}^{-1} z_{2}^{-1}-\frac{1}{5} z_{1}^{-1} z_{2}+\frac{1}{20} z_{1}^{-2}\right)^{2} .
$$

It implies

$$
\begin{gathered}
\gamma\left(z_{1}, z_{2}\right) \gamma\left(z_{1}^{-1}, z_{2}^{-1}\right)=\frac{221}{200}\left\{1-\frac{4}{17}\left(z_{1} z_{2}+z_{1}^{-1} z_{2}^{-1}\right)\right. \\
\left.-\frac{5}{26}\left(z_{1} z_{2}^{-1}+z_{1}^{-1} z_{2}\right)+\frac{10}{221}\left(z_{1}^{2}+z_{1}^{-2}+z_{2}^{2}+z_{2}^{-2}\right)\right\}
\end{gathered}
$$

Hence, the AER of 2-D AR processes implies that the given ACRF is also from a symmetric noncausal 2-D AR model

$$
\begin{aligned}
y_{s, t} & =\frac{4}{17}\left(y_{s-1, t-1}+y_{s+1, t+1}\right)+\frac{5}{26}\left(y_{s-1, t+1}+y_{s-1, t+1}\right) \\
& -\frac{10}{221}\left(y_{s-2, t}+y_{s+2, t}+y_{s, t-2}+y_{s, t+2}\right)+u_{s, t},
\end{aligned}
$$

where $\left\{u_{s, t}\right\}$ is a 2 -D white noise process with variance $\left(\frac{16}{17} \times \frac{25}{26}\right)^{2} \times 0.6597=$ 0.5403 .

It should be noted that even if a causal 2-D AR model has a finite number of parameters, the corresponding symmetric noncausal 2-D AR model may have an infinite number of parameters.

\section{$5 \quad 2-D$ AR Spectrum Estimate}

The ARE of 2-D AR processes and Algorithm A can be utilized to 2-D spectral analysis. Let $\left\{y_{s, t} \mid s=1,2, \cdots, S, t=1,2, \cdots, T\right\}$ be an $S T$-realization of a second-order stationary random field. Then, the ACVF and the ACRF 
are estimated by the sample ACVF and the sample ACRF, respectively, which are defined as

$$
\begin{gathered}
\hat{\sigma}(j, k)=\hat{\sigma}(-j,-k)=\frac{1}{(S-j)(T-k)} \sum_{s=1}^{S-j} \sum_{t=1}^{T-k}\left\{y_{s+j, t+k}-\bar{y}\right\}\left\{y_{s, t}-\bar{y}\right\} \\
(j, k=0,1, \cdots) \\
\hat{\sigma}(j,-k)=\hat{\sigma}(-j, k)=\frac{1}{(S-j)(T-k)} \sum_{s=1}^{S-j} \sum_{t=k+1}^{T}\left\{y_{s+j, t-k}-\bar{y}\right\}\left\{y_{s, t}-\bar{y}\right\} \\
(j, k=0,1, \cdots) \\
\hat{\rho}(j, k)=\hat{\sigma}(j, k) / \hat{\sigma}(0,0), \quad(j, k=0, \pm 1, \pm 2, \cdots)
\end{gathered}
$$

where $\bar{y}$ is the sample mean. The sample ACVF and the sample ACRF are unbiased-type estimators. It should be noted that a biased-type estimator of either the ACVF or the ACRF is inconsistent and does not have an asymptotic distribution [31], [32]. Substituting the sample ACVF for the ACVF in the 2-D Yule-Walker equations and solving them using Algorithm A, we obtain the 2-D Yule-Walker estimates.

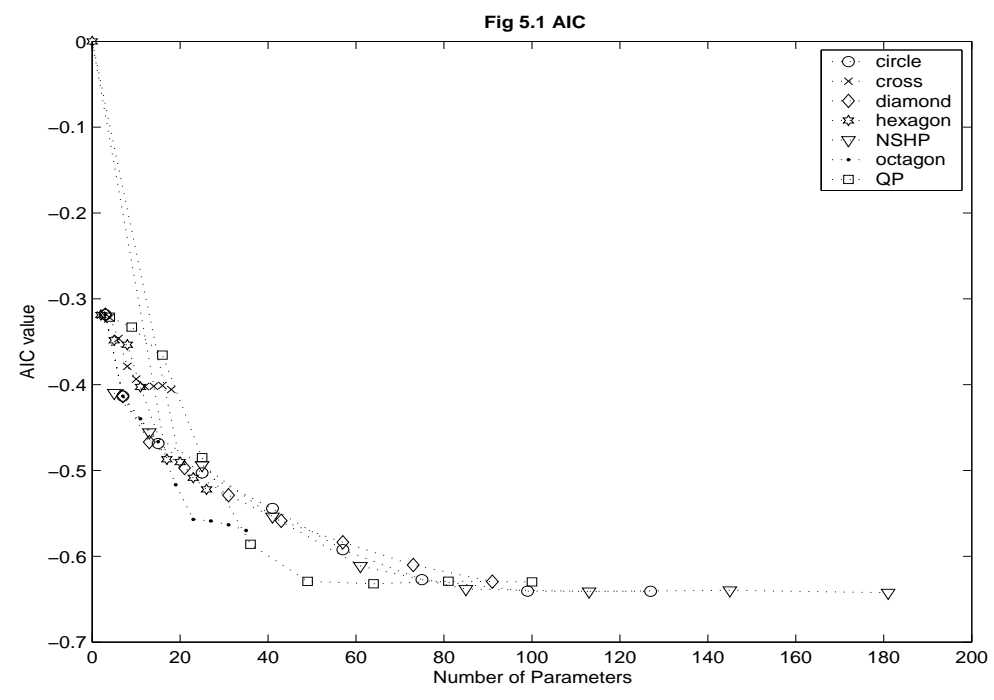


In a practical situation, we do not know the true order $p=p_{0}$ of the ROS $G_{p}$ of a $2-\mathrm{D}$ AR process, even though we assume it exists. Thus, it is necessary to estimate $p_{0}$. As in 1-D AR model identification, we can calibrate the true order $p_{0}$ using the 2-D Yule-Walker estimates and some penalty function methods such as the AIC, the BIC and $\phi_{\beta}$ [22], [33], which are defined by

$$
\begin{aligned}
& A I C(m)=\log \sigma_{G_{m}}^{2}+\frac{2}{S T}\{S(m)+1\}, \\
& B I C(m)=\log \sigma_{G_{m}}^{2}+\frac{\log (S T)}{S T}\{S(m)+1\}, \\
& \phi_{\beta}(m)=\log \sigma_{G_{m}}^{2}+\frac{(S T)^{\beta} \log \log (S T)}{S T}\{S(m)+1\}
\end{aligned}
$$

with $\beta=\frac{\log \log (S T)}{\log (S T)}$. We may also use the minimum eigenvalue criterion $(\mathrm{MEV})$, which is asymptotically equivalent to the BIC [34]. Since we can easily calculate white-noise variances $\left\{\sigma_{G_{m}}^{2} \mid m=1,2, \cdots\right\}$ using Algorithm A, we prefer the BIC to the MEV that needs eigenvalues of large matrices.

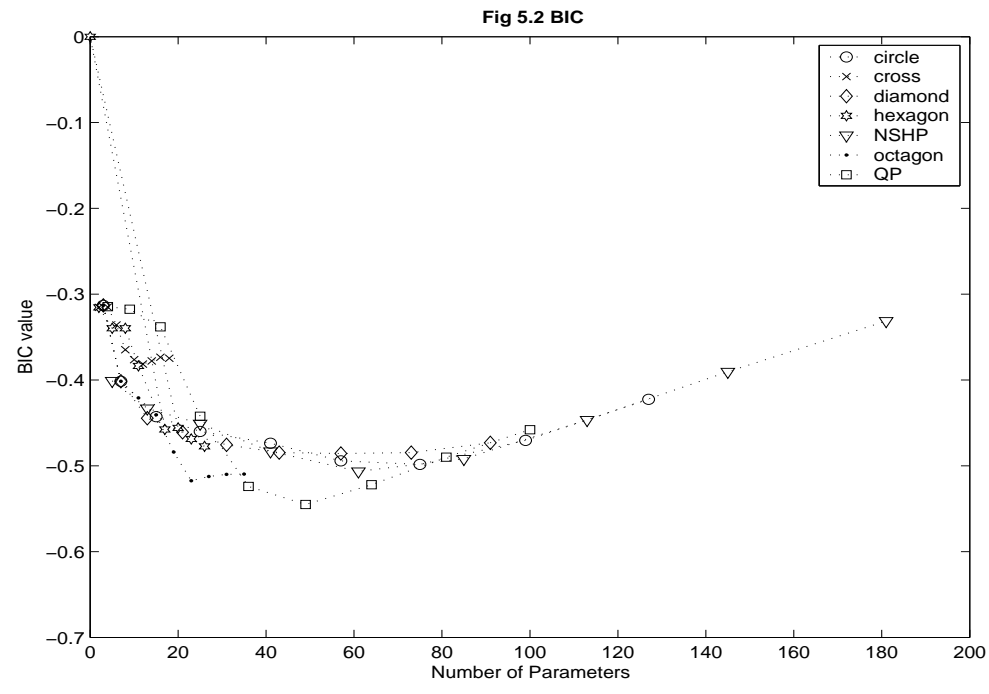


To illustrate the usefulness of the ARE and Algorithm A in 2-D AR spectrum estimation, we reconsider the following example given in [26].

(Example 5.1) Generate a $60 \times 60$ array $\left\{y_{s, t}\right\}$ from a sinusoidal model

$$
y_{s, t}=\cos \left(\frac{\pi s}{5}+\frac{\pi t}{2}\right)+\cos \left(\frac{\pi s}{10}-\frac{\pi t}{10}\right)+v_{s, t},
$$

where $\left\{v_{s, t}\right\}$ is a $2-\mathrm{D}$ white-noise process with variance 1 . Firstly, we calculate sample autocorrelations $\{\hat{\rho}(j, k)\}$. Secondly, applying Algorithm A to the sample ACRF, we calculate the AIC, the BIC and $\phi_{\beta}$ of $2-\mathrm{D}$ AR models with half-cross, half-diamond, QP-square, half-square, half-hexagon, half-octagon, and half-circle $\operatorname{ROS} G_{p}$ for $p=1,2, \cdots, 9$. Their values are depicted in Fig 5.1, Fig 5.2, and Fig 5.3, respectively. As shown in Fig 5.1, the AIC value decreases as the number of parameters increases. It means the AIC has a strong tendency to overidentify a 2-D AR model. As shown in Fig 5.2, minimum values of the BIC are near between -5.1 and -5.5, which are obtained by 2-D AR models with QP-square ROS of 36, 49, and 64 parameters, and with half-octagon ROS of $23,27,31$, and 35 parameters. The BIC value of a QP-square ROS of 49 parameters is -0.5450 , and that of a half-octagon ROS of 23 parameters is -0.5175 . Even though the difference of parameter numbers is 26 , the difference in BIC values is only 0.0275 . Therefore, the principle of parsimony leads us to prefer the latter. As shown in Fig 5.3, the 2-D AR model with a half-octagon ROS of 23 parameters has the minimum $\phi_{\beta}$ value -0.4598 . Thus, we select it as a proper model to the given realization. It should be noted that Nakachi et al. [26] estimate the spectrum using a 2-D AR model with a half-circle ROS of 60 parameters. 
Its $\mathrm{BIC}$ and $\phi_{\beta}$ values are around -0.49 and -0.35 , respectively, and they are larger than those of the selected 2-D AR model with a half-octagon ROS with 23 parameters.

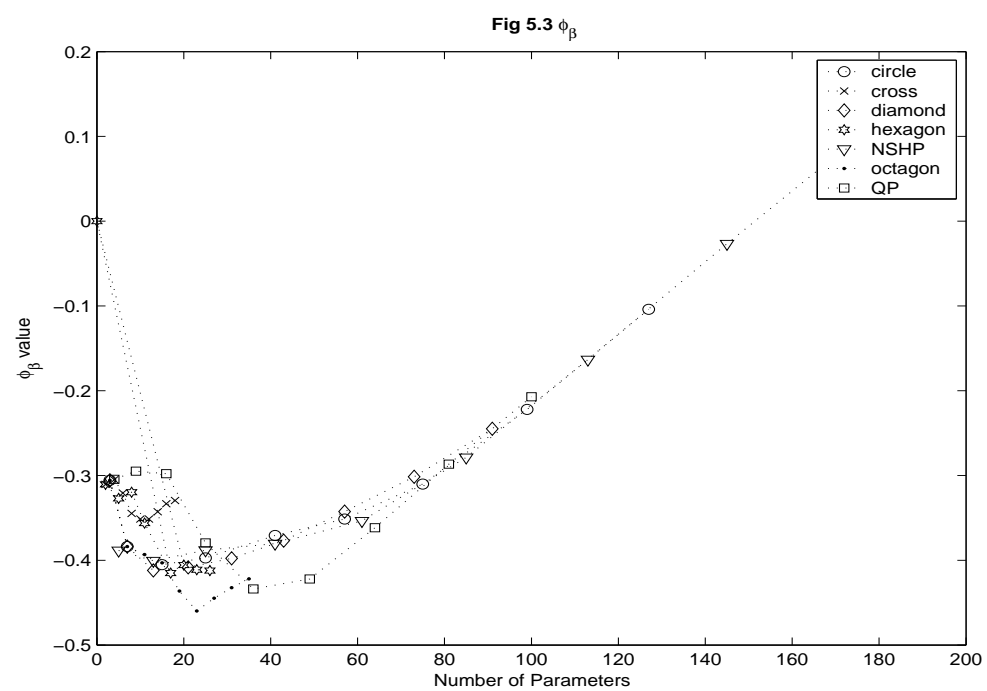

Fig 5.4 shows the estimated spectrum of the selected 2-D AR model with a half-octagon ROS of 23 parameters, and its contour map is in Fig 5.5. They show four prominent spectral peaks accurately.

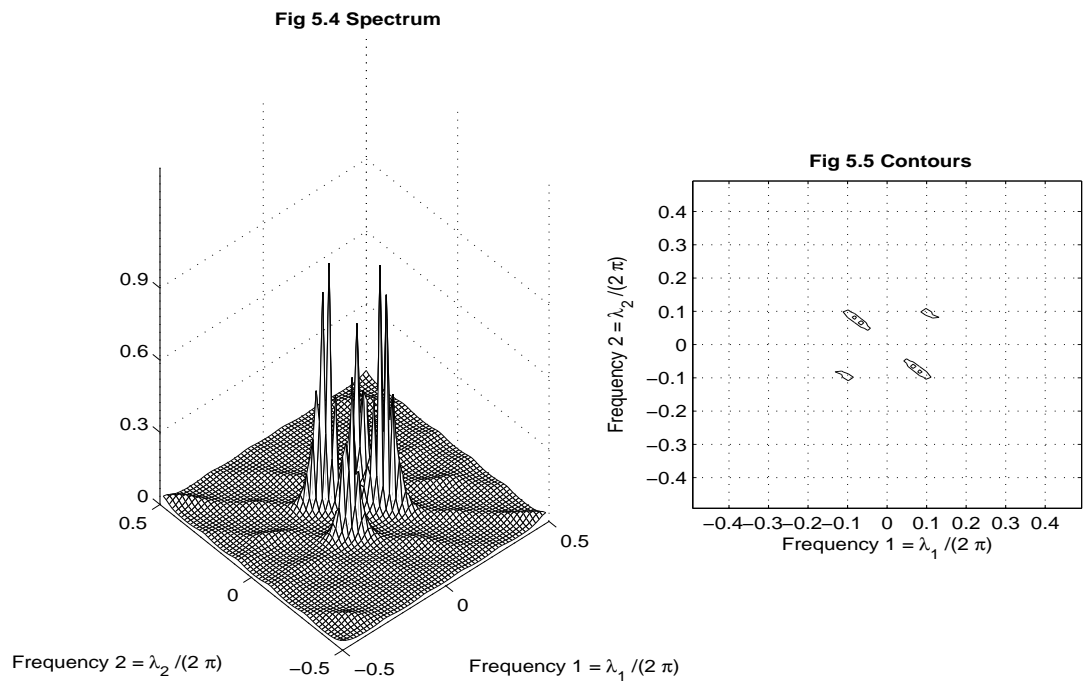




\section{Comments}

Using the AER of 2-D AR processes and Algorithm A, we can specify a noncausal 2-D AR process through a causal 2-D AR model. Therefore, it is not necessary to develop new modeling theories for noncausal 2-D AR processes such as maximum likelihood estimation, order determination methods and diagnostic checking methods, which are difficult to accomplish. Instead, we study causal 2-D AR models with various ROS, and utilize the results in noncausal 2-D AR modeling. The results in this paper can be extended to 3-D AR processes using the methods of [35].

\section{Appendix}

We are going to derive ACRF's of the 2-D AR processes discussed in Sections 3 and 4 . Since $\sigma(-j,-k)=\sigma(j, k)$, we consider only the case $j \geq 0$. Also, assume that $\left\{v_{s, t}\right\}$ is a 2 -D white noise process with variance $\sigma^{2}$.

Firstly, consider a causal 2-D AR process with a half-cross ROS

$$
y_{s, t}=\theta y_{s, t-1}+\theta y_{s-1, t}+v_{s, t}
$$

where $|\theta|<\frac{1}{2}$. The 2-D AR process (40) can be represented by

$$
y_{s, t}=\left\{1-\theta\left(B_{1}+B_{2}\right)\right\}^{-1} v_{s, t}=\sum_{a=0}^{\infty} \sum_{b=0}^{a} \theta^{a}\left(\begin{array}{l}
a \\
b
\end{array}\right) v_{s-b, t-a+b} .
$$

It can be verified using (40) and (41) that

$$
\sigma(j, k)=\sigma^{2} \sum_{a=a_{0}}^{\infty} \sum_{b=0}^{b_{0}}\left(\begin{array}{l}
a \\
b
\end{array}\right)\left(\begin{array}{c}
a+j+k \\
b+j
\end{array}\right) \theta^{2 a+k+j},
$$


where $a_{0}=\max (0,-j-k)$ and $b_{0}=\max (a, a+k)$. For $j+k \geq 0$,

becomes

$$
\sigma(j, k)=\sigma^{2} \sum_{a=0}^{\infty} \sum_{b=0}^{b_{0}}\left(\begin{array}{l}
a \\
b
\end{array}\right)\left(\begin{array}{l}
a+j+k \\
a-b+k
\end{array}\right) \theta^{2 a+k+j}=F_{\theta}(j, k)
$$

where

$$
F_{\theta}(j, k)=\sigma^{2} \sum_{a=0}^{\infty}\left(\begin{array}{c}
2 a+j+k \\
a+k
\end{array}\right) \theta^{2 a+k+j}
$$

For $j+k<0$, it can be verified by putting $f=a+j+k$ in (42) that

$$
\sigma(j, k)=F_{\theta}(-j,-k)
$$

Combining (43) and (44) results in

$$
\rho(j, k)=\rho(-j,-k)= \begin{cases}\frac{1}{F_{\theta}(0,0)} F_{\theta}(j, k), & (j+k \geq 0), \\ \frac{1}{F_{\theta}(0,0)} F_{\theta}(-j,-k), & (-j-k \geq 0) .\end{cases}
$$

Secondly, consider a causal 2-D AR process with a half-diamond ROS

$$
y_{s, t}=\theta_{1,1} y_{s-1, t-1}+\theta_{1,-1} y_{s-1, t+1}-\theta_{1,1} \theta_{1,-1} y_{s-2, t}+v_{s, t},
$$

where $\left|\theta_{1,1}\right|<1$ and $\left|\theta_{1,-1}\right|<1$. The 2-D AR process (46) can be represented by

$y_{s, t}=\left(1-\theta_{1,1} B_{1} B_{2}\right)^{-1}\left(1-\theta_{1,-1} B_{1} B_{2}^{-1}\right)^{-1} v_{s, t}=\sum_{a=0}^{\infty} \sum_{b=0}^{\infty} \theta_{1,1}^{a} \theta_{1,-1}^{b} v_{s-a-b, t-a+b}$

If either $k+j$ or $j-k$ is not even, then (47) implies

$$
\sigma(j, k)=0
$$

Otherwise, it can be verified using (46) and (47) that

$$
\sigma(j, k)=\sigma^{2} \sum_{a=a_{0}}^{\infty} \sum_{b=b_{0}}^{\infty} \theta_{1,1}^{2 a+\frac{k+j}{2}} \theta_{1,-1}^{2 b+\frac{j-k}{2}}
$$


where $a_{0}=\max \left(0,-\frac{j+k}{2}\right)$ and $b_{0}=\max \left(0, \frac{k-j}{2}\right)$. For $k \geq j \geq 0$, (49) becomes

$$
\sigma(j, k)=\sigma^{2} \frac{1}{1-\theta_{1,1}^{2}} \frac{1}{1-\theta_{1,-1}^{2}} \theta_{1,1}^{\frac{k+j}{2}} \theta_{1,-1}^{\frac{k-j}{2}} .
$$

For $-j<k<j,(49)$ becomes

$$
\sigma(j, k)=\sigma^{2} \frac{1}{1-\theta_{1,1}^{2}} \frac{1}{1-\theta_{1,-1}^{2}} \theta_{1,1}^{\frac{k+j}{2}} \theta_{1,-1}^{\frac{j-k}{2}}
$$

For $k \leq-j$, (49) becomes

$$
\sigma(j, k)=\sigma^{2} \frac{1}{1-\theta_{1,1}^{2}} \frac{1}{1-\theta_{1,-1}^{2}} \theta_{1,1}^{-\frac{k+j}{2}} \theta_{1,-1}^{\frac{j-k}{2}}
$$

Combining (50)-(52) results in

$$
\rho(j, k)=\rho(-j,-k)=\theta_{1,1}^{\left|\frac{k+j}{2}\right|} \theta_{1,-1}^{\left|\frac{j-k}{2}\right|}, \quad(j=0,1, \cdots),
$$

if both $k+j$ and $j-k$ are even. Otherwise, $\rho(j, k)=\rho(-j,-k)=0$.

Thirdly, consider a 2-D AR process with a half-diamond ROS

$$
\begin{aligned}
y_{s, t} & =\theta_{1,1} y_{s-1, t-1}+\theta_{1,-1} y_{s-1, t+1}-\theta_{1,1}^{2} y_{s-2, t-2}-3 \theta_{1,1} \theta_{1,-1} y_{s-2, t} \\
& -\theta_{1,-1}^{2} y_{s-2, t+2}+2 \theta_{1,1}^{2} \theta_{1,-1} y_{s-3, t-1}+2 \theta_{1,1} \theta_{1,-1}^{2} y_{s-3, t+1} \\
& -\theta_{1,1}^{2} \theta_{1,-1}^{2} y_{s-4, t}+v_{s, t}
\end{aligned}
$$

where $\left|\theta_{1,1}\right|<1$ and $\left|\theta_{1,-1}\right|<1$. The 2-D AR process (54) can be represented by

$$
\begin{aligned}
y_{s, t} & =\left(1-\theta_{1,1} B_{1} B_{2}\right)^{-2}\left(1-\theta_{1,-1} B_{1} B_{2}^{-1}\right)^{-2} v_{s, t} \\
& =\sum_{a=0}^{\infty} \sum_{b=0}^{\infty}(a+1)(b+1) \theta_{1,1}^{a} \theta_{1,-1}^{b} v_{s-a-b, t-a+b} .
\end{aligned}
$$


If either $k+j$ or $j-k$ is not even, then (55) implies

$$
\sigma(j, k)=0
$$

Otherwise, it can be verified using (54) and (55) that

$$
\sigma(j, k)=\sigma^{2} \sum_{a=a_{0}}^{\infty} \sum_{b=b_{0}}^{\infty} a\left(a+\frac{k+j}{2}\right) \theta_{1,1}^{2 a+\frac{k+j}{2}} b\left(b+\frac{j-k}{2}\right) \theta_{1,-1}^{2 b+\frac{j-k}{2}}
$$

where $a_{0}$ and $b_{0}$ are the same as before. Let $g(\nu, l)=\sum_{a=0}^{\infty} a(a+l) \nu^{2 a+l}$. It can be easily shown that

$$
\begin{aligned}
g(\nu, l) & =\sum_{a=1}^{\infty} a(a+l) \nu^{2 a+l} \\
& =\nu^{l+2} \sum_{a=1}^{\infty} a(a+1)\left(\nu^{2}\right)^{a-1}+(l-1) \nu^{l+2} \sum_{a=1}^{\infty} a\left(\nu^{2}\right)^{a-1} \\
& =\frac{\nu^{l+2}}{\left(1-\nu^{2}\right)^{3}}\left\{2+(l-1)\left(1-\nu^{2}\right)\right\}
\end{aligned}
$$

For $k \geq j \geq 0,(57)$ becomes

$$
\sigma(j, k)=\sigma^{2} g\left(\theta_{1,1}, \frac{j+k}{2}\right) g\left(\theta_{1,-1}, \frac{k-j}{2}\right) .
$$

For $-j<k<j,(57)$ becomes

$$
\sigma(j, k)=\sigma^{2} g\left(\theta_{1,1}, \frac{j+k}{2}\right) g\left(\theta_{1,-1}, \frac{j-k}{2}\right) .
$$

For $k \leq-j$, (57) becomes

$$
\sigma(j, k)=\sigma^{2} g\left(\theta_{1,1},-\frac{j+k}{2}\right) g\left(\theta_{1,-1}, \frac{j-k}{2}\right) .
$$

Combining (59)-(61) results in

$\rho(j, k)=\rho(-j,-k)=\frac{1}{g\left(\theta_{1,1}, 0\right) g\left(\theta_{1,-1}, 0\right)} g\left(\theta_{1,1},\left|\frac{j+k}{2}\right|\right) g\left(\theta_{1,-1},\left|\frac{j-k}{2}\right|\right)$, 
if both $k+j$ and $j-k$ are even. Otherwise, $\rho(j, k)=\rho(-j,-k)=0$.

Fourthly, consider a causal 2-D AR process in the NSHP,

$$
y_{s, t}=\theta_{1} y_{s, t-r}+\theta_{-1} y_{s-p, t+q}-\theta_{1} \theta_{-1} y_{s-p, t-r+q}+v_{s, t},
$$

where $p, q$ and $r$ are positive integers, $\left|\theta_{1}\right|<1$ and $\left|\theta_{-1}\right|<1$. The 2-D AR process (63) can be represented by

$$
y_{s, t}=\left(1-\theta_{1} B_{2}^{r}\right)^{-1}\left(1-\theta_{-1} B_{1}^{p} B_{2}^{-q}\right)^{-1} v_{s, t}=\sum_{a=0}^{\infty} \sum_{b=0}^{\infty} \theta_{1}^{a} \theta_{-1}^{b} v_{s-p b, t-r a+q b} .
$$

Let $l=(j q+k p) /(r p)$ and $a_{0}=\max (0,-l)$. If either $j / p$ or $l$ is not an integer, then it can be verified using (64) that

$$
\sigma(j, k)=0
$$

If both $j / p$ and $l$ are nonnegative integers, it can be verified using (63) and (64) that

$$
\sigma(j, k)=\sigma^{2} \sum_{a=0}^{\infty} \sum_{b=0}^{\infty} \theta_{1}^{2 a+l} \theta_{-1}^{2 b+j / p}=\sigma^{2} \frac{1}{1-\theta_{1}^{2}} \theta_{1}^{l} \frac{1}{1-\theta_{-1}^{2}} \theta_{-1}^{j / p} .
$$

If both $j / p$ and $-l$ are positive integers, it can be verified using (63) and (64) that

$$
\sigma(j, k)=\sigma^{2} \sum_{a=-l}^{\infty} \sum_{b=0}^{\infty} \theta_{1}^{2 a+l} \theta_{-1}^{2 b+j / p}=\sigma^{2} \frac{1}{1-\theta_{1}^{2}} \theta_{1}^{-l} \frac{1}{1-\theta_{-1}^{2}} \theta_{-1}^{j / p} .
$$

Combining (66) and (67) results in

$$
\rho(j, k)=\rho(-j,-k)=\theta_{1}^{\mid \frac{j q+k p}{r p}} \mid \theta_{-1}^{\left|\frac{j}{p}\right|}, \quad(j=0,1, \cdots),
$$

if both $l$ and $j / p$ are integers. Otherwise, $\rho(j, k)=\rho(-j,-k)=0$. 


\section{References}

[1] R. L. Kashyap and R. Chellappa, "Estimation and choice of neighbors in spatial-interaction models of images," IEEE Trans. Information Theory, vol. IT-29, no. 1, pp. 60-72, 1983.

[2] H. Kaufman and A. M. Tekalp, "Survey of estimation techniques in image restoration," IEEE Control Systems Magazine, vol. 11, no, 1, pp. 16-24, 1991.

[3] S. Oe, "Texture segmentation method using two-dimensional AR model and Kullback information," Pattern Recognition, vol. 26, no, 12, pp. 237-244, 1993.

[4] H. Mhidra, J. Brochard and M. Leard, "AR models and bidimensional discrete moments applied to texture modeling and recognition," Pattern Recognition, vol. 26, no, 5, pp. 721-726, 1993.

[5] S. W. Lu and H. Xu, "Textured image segmentation using autoregressive model and artificial neural network," Pattern Recognition, vol. 28, no, 12, pp. 1807-1817, 1995.

[6] A. Sarkar, K. M. S. Sharma and R. V. Sonak, "A new approach for subset 2-D AR model identification for describing textures," IEEE Trans. Image Proc., vol. 6, pp. 407-413, 1997.

[7] F. Heitz, H. Maitre and C. de Couessian, "Application of autoregressive models to fine arts painting analysis," Signal Processing, vol. 13, pp. $1-14,1987$.

[8] G. Sharma and R. Chellappa, "Two-dimensional spectrum estimation using noncausal autoregressive models," IEEE Trans. Information Theory, vol. IT-32, No. 2, pp. 268-275, 1986.

[9] R. R. Hansen and R. Chellappa, "Noncausal 2-D spectrum estimation for direction finding," IEEE Trans. Information Theory, vol. IT-36, No. 1, pp. 108-125, 1990. 
[10] D. N. Politis, "A simple information theoretic proof of the maximum entropy property of some Gaussian random fields," IEEE Trans. Image Proc., vol. 3, no. 6, pp. 865-868, 1994.

[11] J. Besag, "Spatial interaction and the statistical analysis of lattice systems (with discussions)," J. Roy. Statist. Soc., Ser. B, vol. 36, pp. 192-236, 1974.

[12] D. E. Dudgeon and R. M. Mersereau, Multidimensional Digital Signal Processing, Englewood Cliffs: Prentice Hall, Inc., 1984.

[13] H. Q. Lu and L. F. Chaparro, "Recursive solution and stability conditions for 2-D least-squares modeling and stabilization problems," Journal of the Franklin Institute, vol. 327, issue 2, pp. 259-271, 1990.

[14] J. V. Krogmeier and K. S. Arun, "On the recursive computation of interpolators with nonrectangular masks," IEEE Trans. Signal Processing, vol. 44, No. 5, pp. 1072-1079,1996,

[15] G. O. Glentis, C. H. Slump and O. E. Herrmann, "A versatile algorithm for two-dimensional symmetric noncausal modeling," IEEE Trans. Circuits and Systems-II: Analog and Digital Signal Processing, vol. 45, no. 2, pp. 251-256, 1998.

[16] G. O. Glentis, C. H. Slump and O. E. Herrmann, "A true order recursive algorithm for two-dimensional mean squared error liner prediction and filtering," Signal Processing, vol. 80, pp. 1399-1418, 2001.

[17] K. Ord, "Estimation methods for models of spatial interaction," $J$. Amer. Stat. Assoc., vol. 70, pp. 120-126, 1975.

[18] P. Y. Zhao and D. R. Yu, "An unbiased and computationally efficient LS estimation method for identifying parameters of 2-D noncausal SAR models," IEEE Trans. Signal Processing, vol. 41, no. 2, pp. 849-857, 1993. 
[19] B. S. Choi, "Model identification of a noncausal 2-D AR Process using a causal 2-D AR model on the nonsymmetric half-plane," IEEE Trans. Signal Processing, vol. 51, no. 5, pp. 1412-1421, 2003.

[20] B. S. Choi, "A fundamental theorem of algebra, spectral factorization, and stability of 2-D Systems," IEEE Trans. Signal Processing, vol. 51, no. 3, pp. 853-863, 2003.

[21] O. Alata, M. Najim and C. Ramananjarasosa, "Extension of the ShurCohen stability test for 2-D AR quarter-plane model," IEEE Trans. Information Theory, vol. 49, No. 11, pp. 3099-3106, 2003.

[22] B. S. Choi, ARMA Model Identification, New York: Springer-Verlag, 1992.

[23] P. Whittle, "On stationary processes in the plane," Biometrika, vol. 41, pp. 434-449, 1954.

[24] J. E. Besag, "On the correlation structure of some two-dimensional stationary processes," Biometrika, vol. 59, pp. 43-48, 1972.

[25] H. Lev-Ari, S. R. Parker and T. Kailath, "Multidimensional maximumentropy covariance extension," IEEE Trans. Information Theory, vol. 35, No. 3, pp. 497-508, 1989.

[26] T. Nakachi, K. Yamashita, and N. Hamada, "Asymmetric half-plane lattice modeling based on 2-D Levinson algorithm," IEEE Trans. Circuits and Systems-II: Analog and Digital Signal Processing, vol. 44, no. 10, pp. 865-868, 1997.

[27] B. S. Choi, "A recursive algorithm for solving the spatial Yule-Walker equations of causal spatial AR models," Stat. 85 Prob. Letters, vol. 33, pp. 241-251, 1997.

[28] B. S. Choi, "A recursive algorithm for solving the 3-D Yule-Walker equations of causal 3-D AR models," IEEE Trans. Signal Processing, vol. 47, 1999, pp. 2491-2502. 
[29] M. Wax and T. Kailath, "Efficient inversion of Toeplitz-block Toeplitz matrix," IEEE Trans. Acoustics, Speech, and Signal Processing, vol. ASSP-31, no. 5, pp. 1218-1221, 1983.

[30] N. Kalouptsidis, G. Carayannis, and D. Manolakis, "Fast algorithms for block Toeplitz matrices with Toeplitz entries," Signal Processing, vol. 6, pp. 77-81, 1984.

[31] X. Guyon, "Parameter estimation for a stationary process on a $d$ dimensional lattice," Biometrika, vol. 69, pp. 95-105, 1982.

[32] B. S. Choi, "On the asymptotic distributions of mean, autocovariance, autocorrelation, crosscovariance and impulse response estimators of a stationary multidimensional random field," Commun. Statist.-Theory Meth., vol. 29, pp. 1703-1724, 2000.

[33] O. Alata and C. Olivier, "Choice of a 2-D causal autoregressive texture model using information criteria," Pattern Recognition Letters, vol. 24, pp. 1191-1201, 2003.

[34] B. Aksasse and L. Radouane, "Two-dimensional autoregressive (2-D AR) model order estimation," IEEE Trans. Signal Processing, vol. 47, no. 7, pp. 2072-2077, 1999.

[35] B. S. Choi, "Model specification of a noncausal 3-D AR process using a causal 3-D AR model on the nonsymmetric half-space," Multidimensional Systems and Signal Processing, vol. 14, pp. 319-341, 2003. 